Article

\title{
On the Polynomial Solutions and Limit Cycles of Some Generalized Polynomial Ordinary Differential Equations
}

\author{
Claudia Valls
}

Departamento de Matemática, Instituto Superior Técnico, Universidade de Lisboa, 1049-001 Lisboa, Portugal; cvalls@math.tecnico.ulisboa.pt

Received: 6 July 2020; Accepted: 9 July 2020; Published: 12 July 2020

check for updates

\begin{abstract}
We study equations of the form $y d y / d x=P(x, y)$ where $P(x, y) \in \mathbb{R}[x, y]$ with degree $n$ in the $y$-variable. We prove that this ordinary differential equation has at most $n$ polynomial solutions (not necessarily constant but coprime among each other) and this bound is sharp. We also consider polynomial limit cycles and their multiplicity.
\end{abstract}

Keywords: polynomial ordinary differential equations; polynomial solutions

\section{Introduction}

Consider the ordinary differential equation

$$
y \frac{d y}{d x}=A_{0}(x)+A_{1}(x) y+\ldots+A_{n}(x) y^{n}
$$

where $x, y$ are real variables, $A_{i}(x) \in \mathbb{R}[x]$ for $i=0,1, \ldots, n$ and $n \in \mathbb{N}$ with $n \geq 1$ ( $\mathbb{R}[x]$ is the set of polynomials with real coefficients). We also assume that $A_{0} A_{n} \not \equiv 0$. The derivative of $y$ with respect to $x$ will be denoted as $d y / d x$ or $y^{\prime}$.

We study the solutions of Equation (1) of the form $y=p(x)$ where $p \in \mathbb{R}[x]$, not necessarily constant (otherwise it is trivial). The computation of solutions (either polynomial or rational) of a nonlinear differential equation has a remarkable role in understanding their dynamical properties and their whole set of solutions. In 1936 it was Rainville in [1] the first one who determined all the Riccati differential equations $y^{\prime}=A_{0}(x)+A_{1}(x) y+y^{2}$, with $A_{0}(x)$ and $A_{1}(x)$ being polynomials that have polynomial solutions. Not only this, he also provided an algorithm for computing these solutions. Campbell and Golomb in [2] gave an algorithm for obtaining all polynomial solutions of the generalized Riccati equation of the form $B(x) y^{\prime}=A_{0}(x)+A_{1}(x) y+A_{2}(x) y^{2}$ where $B, A_{i} \in \mathbb{R}[x]$ for $i=0,1,2$. More recently, Behloul and Cheng in [3] gave another algorithm to detect either polynomial or rational solutions of

$$
B(x) y^{\prime}=A_{0}(x)+A_{1}(x) y+A_{2}(x) y^{2}+\cdots+A_{n}(x) y^{n}
$$

where $B, A_{i}$ are polynomials in $x$ for $i=0, \ldots, n$. Equation (1) was previously studied in [4] where the authors provide an upper bound on the number of polynomial solutions of Equation (1) in terms of $n$ and the degree of $a_{0}(x)$. In particular when this degree is not zero the upper bound is very far from being optimal. Moreover, for some very particular classes and some particular values of $n$ they study properties of their polynomial solutions. In the present paper, with very different techniques, we first provide universal bounds (in the sense that they only depend on $n$ ) on the polynomial solutions of Equation (1) that are much more accurate and we also show that these upper bounds are optimal. 
Second we consider polynomial limit cycles and their multiplicity. We recall that besides fixed points (very well-understood), limit cycles (that is, isolated periodic orbits) are the asymptotic state of other solutions of a given system and thus of great importance for understanding their dynamical properties. Limit cycles are the subject of the so-called Hilbert's 16th problem, which essentially asks for an upper bound, uniformly in terms of the degree $n$, for the maximum number of limit cycles of a planar polynomial vector field. This problem lasts for more than 100 years and all attempts to solve it (all failed) has produced significant progress mainly in the geometric theory of planar vector fields, but also in bifurcation theory, the study of normal forms, foliations and also in some topics in algebraic geometry. Up to now, it is unknown whether such a uniform upper bound exists, even when the degree is two. Solution for Hilbert's 16th problem mostly consist on restricting the classes of vector fields to particular simpler ones (such as our Equation (1)), see e.g., [5] for an overview.

When the left-hand side of Equation (1) is of the form $d y / d x$, there are more studies of either the number of polynomial solutions or of rational solutions. In this easier scenario, the polynomial solutions of these equations have been intensively investigated. In the case when the functions $A_{i}(x)$ are periodic we want to mention the papers [6-15] and the references therein. A special mention is done to the papers [16-19]. In the first two, the authors study the number of polynomial solutions of either polynomial Riccati or polynomial Bernouilli equations, and Abel equations of certain type, and in the third one, the authors obtain the maximum number and the multiplicity of the polynomial limit cycles of $y^{\prime}=A_{0}(x)+A_{1}(x) y+\ldots+A_{n}(x) y^{n}$ where $A_{i} \in \mathbb{R}[x]$ for $i=0, \ldots, n$ and $A_{n}(x) \neq 0$. We also want to mention that the study of the number of rational solutions of either polynomial Riccati or polynomial Bernouilli equations was done in [20] and of Abel equations was done in [21,22].

The following theorem, which is our first main theorem, establishes for the differential Equation (5), the maximum number of coprime polynomial solutions (i.e., $p(x), q(x) \in \mathbb{R}[x]$ satisfying $\operatorname{gcd}\{p(x), q(x)\}=1$ ) and periodic polynomial solutions (i.e., solutions of the form $y=\varphi(x)$ where $\varphi:[0,1] \rightarrow \mathbb{R}$ is $C^{1}$ and satisfy $\left.\varphi(0)=\varphi(1)\right)$. It also establishes the solutions that are the inverse of a polynomial. We recall that the period of a periodic polynomial solution is one but we could choose any period to define a periodic solution because by making an affine change in the variable $x$ one can considers just period one.

Theorem 1. The following statements hold for the polynomial ordinary differential Equation (1).

(a) It has at most $n$ solutions that are constant, and there are examples with exactly $n$ constant solutions.

(b) If $n=1$ it has a polynomial solution which is not constant, then it has infinitely many polynomial solutions.

(c) If $n \geq 2$, the difference between two coprime polynomial solutions is a constant.

(d) If $n \geq 2$, there are examples with $n$ polynomial periodic solutions.

(e) If $n \geq 2$, given $n$ non-constant polynomials coprime among each other, there are equations with such $n$ solutions.

(f) If $n \geq 2$, given $n+1$ non-constant polynomials, coprime among each other, there are no equations with such $n+1$ solutions.

(g) If $n \geq 2$, it has at most $n$ polynomial solutions that are coprime among each other, and there are examples with such n polynomial solutions.

Theorem 1 is proved in Section 2. In the context of the proof of Theorem 1 (see Section 2) in a different but similar exploratory application and in a little broader context, one typical case leading to systems of equations is in multiple linear regressions by maximizing the likelihood under assumption of generalized Gauss-Laplace distribution error. As further exploratory cases one may also want to consider the cases involving characteristic polynomials (see [23]).

From Theorem 1 we have readily the following result. Let $\mathcal{G}$ be the space of $C^{1}$ functions $\varphi:[0,1] \rightarrow \mathbb{R}$ satisfying $\varphi(0)=\varphi(1)$ endowed with the supremum topology. A periodic 
solution $y=\varphi(x)$ of (1) is said to be a limit cycle if there exists in $\mathcal{G}$ a neighborhood of $\varphi$ without any other periodic solution.

Theorem 2. Any ordinary differential Equation (1) has at most $n$ polynomial limit cycles coprime among each other and this bound is reached.

We also study the multiplicity of the differential Equation (1). In order to define the multiplicity we will consider the so-called translation operator. Let $\psi\left(x ; y_{0}\right)$ be the solution of Equation (1) defined for $x \in[0,1]$ such that $\psi\left(0 ; y_{0}\right)=y_{0}$. The function $\Psi: \mathbb{R} \rightarrow \mathbb{R}$ satisfying $\Psi\left(y_{0}\right)=\psi\left(1 ; y_{0}\right)-y_{0}$ is called the translation operator associated with (1). Following Lloyd [24,25], the multiplicity of $y_{0}$ as a zero of $\Psi\left(y_{0}\right)$ is the multiplicity of a limit cycle of (1) associated with the isolated zero $y_{0}$ of the translation operator.

Theorem 3. Let $y=\varphi(x)$ be a periodic solution of Equation (1) and assume that $A_{0}=\varphi^{3} \bar{A}_{0}$ with $\bar{A}_{0} \in \mathbb{R}[x]$. Then $\varphi$ is:

(i) a limit cycle of multiplicity one if and only if $\mathcal{A}_{1}(1) \neq 0$;

(ii) a limit cycle of multiplicity two if and only if $\mathcal{A}_{1}(1)=0$ and $\mathcal{A}_{2}(1) \neq 0$;

(iii) a limit cycle of multiplicity three if and only if $\mathcal{A}_{1}(1)=\mathcal{A}_{2}(1)=0$ and $\mathcal{A}_{3}(1) \neq 0$;

(iv) a limit cycle of multiplicity greater than or equal to four, or it belongs to a continuum of periodic orbits if $\mathcal{A}_{1}(1)=\mathcal{A}_{2}(1)=\mathcal{A}_{3}(1)=0$.

where

$$
\begin{aligned}
& \mathcal{A}_{1}(x)=-\int_{0}^{x}\left(\varphi^{3}(\sigma) \bar{A}_{0}(\sigma)-\frac{\partial \mathcal{F}}{\partial y}(\sigma, \varphi(\sigma))\right) d \sigma, \\
& \mathcal{A}_{2}(x)=\int_{0}^{x} e^{\mathcal{A}_{1}(\sigma)}\left(\varphi^{2}(\sigma) \bar{A}_{0}(\sigma)+\frac{1}{2} \frac{\partial^{2} \mathcal{F}}{\partial y^{2}}(\sigma, \varphi(\sigma))\right) d \sigma,
\end{aligned}
$$

and

$$
\mathcal{A}_{3}(x)=-\int_{0}^{x} e^{2 \mathcal{A}_{1}(\sigma)}\left(\varphi(\sigma) \bar{A}_{0}(\sigma)-\frac{1}{6} \frac{\partial^{3} \mathcal{F}}{\partial y^{3}}(\sigma, \varphi(\sigma))\right) d \sigma
$$

being

$$
\mathcal{F}(x, y)=A_{2}(x) y+A_{3}(x) y^{2}+\cdots+A_{n}(x) y^{n-1} .
$$

The proof of Theorem 3 is given in Section 3.

\section{Proof of Theorem 1}

Let $y=p(x)=\xi$ be a polynomial solution of Equation (1) which is constant. Then the polynomial in $y, A_{0}(x)+A_{1}(x) y+\ldots+A_{n}(x) y^{n}=0$ must be divisible by $y-\xi$ and since, its degree in $y$ is $n$, it has at most $n$ different constant roots. Therefore, there are at most $n$ different constant solutions of Equation (1). Please note that

$$
y \frac{d y}{d x}=(y-1)(y-2) \ldots(y-n)
$$

is of the form (1) and has $n$ constant solutions. Statement (a) is proved.

For statement (b), let $y=p(x)$ be a polynomial solution of Equation (1) which is not a constant, i.e.,

$$
p(x) \frac{d p}{d x}=A_{0}(x)+A_{1}(x) p+\ldots+A_{n}(x) p^{n} .
$$


Since $p$ divides the left-hand side of Equation (4), it divides the right-hand side too, and so, $A_{0}(x)=\bar{A}_{0}(x) p(x)$ for some $\bar{A}_{0}(x) \in \mathbb{R}[x]$. Hence, Equation (1) becomes

$$
\frac{d p}{d x}=\left(\bar{A}_{0}(x)+A_{1}(x)\right)+A_{2}(x) p+\ldots+A_{n}(x) p^{n-1}
$$

If $n=1$, then $A_{j}(x)=0$ for $j \geq 2$ and Equation (5) becomes

$$
\frac{d p}{d x}=\bar{A}_{0}(x)+A_{1}(x)
$$

and so

$$
p(x)=\int\left(\bar{A}_{0}(s)+A_{1}(s)\right) d s+c, \quad c \in \mathbb{R},
$$

which proves statement (b).

Now assume $n \geq 2$ and let $q(x)$ be another solution of (1) coprime with $p(x)$. Then we must have that $A_{0}(x)=q(x) \tilde{A}_{0}(x)$ for some $\tilde{A}_{0}(x) \in \mathbb{R}[x]$. In short, since $p(x)$ and $q(x)$ are coprime we must have $A_{0}(x)=p(x) q(x) \bar{A}_{0}(x)$ with $\bar{A}_{0}(x) \in \mathbb{R}[x]$. We consider the change $w=q-p$ and we transform Equation (5) into the equation

$$
\begin{aligned}
\frac{d w}{d x}= & \frac{d q}{d x}-\frac{d p}{d x}=\bar{A}_{0}(x) p(x)+A_{1}(x)+A_{2}(x) q(x)+\ldots+A_{n}(x) q(x)^{n-1} \\
& -\bar{A}_{0}(x) q(x)-A_{1}(x)-A_{2}(x) p(x)-\ldots-A_{n}(x) p(x)^{n-1} \\
= & \left(-\bar{A}_{0}(x)+A_{2}(x)\right) w+A_{3}(x) q^{2}(x)+\ldots+A_{n}(x) q(x)^{n-1} \\
& -A_{3}(x) p^{2}-\ldots-A_{n}(x) p(x)^{n-1} \\
= & A_{0}^{*}(x) w+A_{2}^{*}(x) w^{2}+\ldots+A_{n}(x) w^{n-1}
\end{aligned}
$$

where each $A_{i}^{*}$ is a polynomial depending on $A_{j}(x)$ and $\bar{A}_{0}(x)$ for $j=i, \ldots, n$ and some powers of $p(x)$, for $i=2, \ldots, n-1$. Let $w=r(x)$ be another polynomial solution of Equation (6) different from $w=0$, i.e.,

$$
r^{\prime}(x)=\tilde{A}_{2}(x) r(x)+\tilde{A}_{3}(x) r(x)^{2}+\ldots+A_{n}(x) r(x)^{n-1} .
$$

Then since $r(x)$ divides the right-hand side of Equation (7), it divides the left-hand side, too. Therefore $r \mid r^{\prime}$ which implies that $r=\xi \in \mathbb{R}$ is a constant. Therefore, any polynomial solution of Equation (6) must be a constant. In short the difference of two coprime polynomial solutions of (5) must be a constant. This proves statement (c).

Since any solution which is constant is also periodic, statement $(\mathrm{d})$ is a direct consequence of statement (c).

To prove statement (e), assume that Equation (5) has $n$ non-constant polynomial solutions $p_{j}(x)$ for $j=1, \ldots, n$ that are coprime among each other. In view of statement (b) we can write $p_{k}(x)=p_{j}(x)+c_{k, j}$ with $c_{k, j} \in \mathbb{R}$ for $k \neq j$ and $j=1, \ldots, n$. We have

$$
p_{j}(x) \frac{d p_{j}}{d x}=A_{0}+A_{1} p_{j}(x)+\cdots+A_{n}(x) p_{j}(x)^{n} .
$$

Since $p_{j}(x)$ divides the left-hand side of Equation (8), it divides $A_{0}(x)$ for any $p_{j}(x)$, $j=1, \ldots, n$. Hence,

$$
A_{0}(x)=\prod_{j=1}^{n} p_{j}(x) \bar{A}_{0}(x),
$$

where $\bar{A}_{0}(x) \in \mathbb{R}[x]$. Therefore, we have the equation

$$
\frac{d p_{j}}{d x}=\prod_{k=1, k \neq j}^{n} p_{k}(x) \bar{A}_{0}+A_{1}(x)+\ldots+A_{n}(x) p_{j}^{n-1}
$$


Since two solutions differ by a constant, we have that

$$
\frac{d p_{j}(x)}{d x}=\frac{d p_{i}(x)}{d x}:=B(x) \text { for any } j \neq i
$$

So we have the following system of $n$ equations with the unknowns $\bar{A}_{0}, A_{1}, \ldots, A_{n}$

$$
B(x)\left(\begin{array}{c}
1 \\
1 \\
\vdots \\
1
\end{array}\right)-\bar{A}_{0}\left(\begin{array}{c}
\prod_{j=2}^{n} p_{j}(x) \\
\prod_{j=1, j \neq 2}^{n} p_{j}(x) \\
\vdots \\
\prod_{j=1}^{n-1} p_{j}(x)
\end{array}\right)=M\left(\begin{array}{c}
A_{1}(x) \\
A_{2}(x) \\
\vdots \\
A_{n}(x)
\end{array}\right)
$$

where $M$ is the matrix

$$
M=\left(\begin{array}{ccccc}
1 & p_{1}(x) & p_{1}(x)^{2} & \cdots & p_{1}(x)^{n-1} \\
1 & p_{2}(x) & p_{2}(x)^{2} & \cdots & p_{2}(x)^{n-1} \\
\vdots & \vdots & \vdots & \ddots & \vdots \\
1 & p_{n}(x) & p_{n}(x)^{2} & \cdots & p_{n}(x)^{n-1}
\end{array}\right)
$$

Hence

$$
\left(\begin{array}{c}
A_{1}(x) \\
A_{2}(x) \\
\vdots \\
A_{n}(x)
\end{array}\right)=M^{-1}\left(B(x)\left(\begin{array}{c}
1 \\
1 \\
\vdots \\
1
\end{array}\right)-\bar{A}_{0}\left(\begin{array}{c}
\prod_{j=2}^{n} p_{j}(x) \\
\prod_{j=1, j \neq 2}^{n} p_{j}(x) \\
\vdots \\
\prod_{j=1}^{n-1} p_{j}(x)
\end{array}\right)\right) .
$$

We claim that the solutions $A_{1}(x), \ldots, A_{n}(x)$ of Equation (9) are polynomials.

To prove the claim, we will first show that $\operatorname{det} M$ is a constant. Please note that $M$ is the Vandermonde matrix

$$
V=\left(\begin{array}{ccccc}
1 & \alpha_{1} & \alpha_{1}^{2} & \ldots & \alpha_{1}^{n-1} \\
1 & \alpha_{2} & \alpha_{2}^{2} & \ldots & \alpha_{2}^{n-1} \\
\vdots & \vdots & \vdots & \ddots & \vdots \\
1 & \alpha_{n} & \alpha_{n}^{2} & \ldots & \alpha_{n}^{-1}
\end{array}\right)
$$

with

$$
\alpha_{i}=p_{i}(x), \quad i=1, \ldots, n
$$

Then we have that

$$
\begin{aligned}
\operatorname{det} M & =(-1)^{n(n-1) / 2} \prod_{1 \leq i<j \leq n}\left(\alpha_{i}-\alpha_{j}\right) \\
& =(-1)^{n(n-1) / 2} \prod_{1 \leq i<j \leq n}\left(p_{i}(x)-p_{j}(x)\right) \\
& =(-1)^{n(n-1) / 2} \prod_{1 \leq i<j \leq n} c_{i, j} \in \mathbb{R} .
\end{aligned}
$$

Let now

$$
R_{i, j}=\left\{\left(m_{1}, \ldots, m_{n-i}\right): 1 \leq m_{1}<\cdots<m_{n-i} \leq n, m_{1}, \ldots, m_{n-i} \neq j\right\}
$$

Again, taking into account that $M$ is the Vandermonde matrix, its inverse $M^{-1}=\left(m_{i, j}\right)_{1 \leq i \leq j}$ is 


$$
\begin{aligned}
m_{i, j} & = \begin{cases}\frac{(-1)^{i-1}}{\prod_{1 \leq m \leq n, m \neq j}\left(p_{m}-p_{j}\right)} \sum_{R_{i j}} p_{m_{1}} \cdots p_{m_{n-i},} & 1 \leq i<n, \\
\frac{1}{\prod_{1 \leq k \leq n, k \neq j}\left(p_{j}-p_{k}\right)}, & i=n\end{cases} \\
& = \begin{cases}\frac{(-1)^{i-1}}{\prod_{1 \leq m \leq n, m \neq j} c_{m, j}} \sum_{R_{i j}} p_{m_{1}} \cdots p_{m_{n-i}}, & 1 \leq i<n, \\
\frac{1}{\prod_{1 \leq k \leq n, k \neq j}\left(p_{j}-p_{k}\right)}, & i=n .\end{cases}
\end{aligned}
$$

Therefore, in view of (9), we have a polynomial solution in $\left(A_{1}(x), \ldots, A_{n}(x)\right)$ for any polynomial $\bar{A}_{0}(x)$. This proves statement (e).

Now we prove statement (f). In this case we will show that there is exactly one set of polynomials $\left(\bar{A}_{0}(x), A_{1}(x), \ldots, A_{n}(x)\right)$ satisfying

$$
\left(\begin{array}{c}
\bar{A}_{0} \\
A_{1} \\
\vdots \\
A_{n}(x)
\end{array}\right)=M_{1}^{-1} B(x)\left(\begin{array}{c}
1 \\
1 \\
\vdots \\
1
\end{array}\right)
$$

where

$$
M_{1}=\left(\begin{array}{cccccc}
\prod_{k=2}^{n+1} p_{k}(x) & 1 & p_{1}(x) & p_{1}(x)^{2} & \cdots & p_{1}(x)^{n-1} \\
\prod_{k=1, k \neq 2}^{n+1} p_{k}(x) & 1 & p_{2}(x) & p_{2}(x)^{2} & \cdots & p_{2}(x)^{n-1} \\
\vdots & \vdots & \vdots & \vdots & \ddots & \vdots \\
\prod_{k=1}^{n} p_{k}(x) & 1 & p_{n+1}(x) & p_{n+1}(x)^{2} & \cdots & p_{n+1}(x)^{n-1}
\end{array}\right)
$$

and that for this unique set of polynomials we have $\bar{A}_{0}=0$ (and so $A_{0}=0$ ) which is not possible. Let

$$
P_{j}^{(\ell)}=\prod_{k=\ell, k \neq j}^{n+1} p_{k}(x) \text { and } P_{j_{1}, j_{2}}^{(\ell)}=\prod_{k=\ell, k \neq j_{1}, k \neq j_{2}}^{n+1} p_{k}(x) .
$$

Then we can write

$$
M_{1}=\left(\begin{array}{cccccc}
P_{1}^{(1)} & 1 & p_{1} & p_{1}^{2} & \cdots & p_{1}^{n-1} \\
P_{2}^{(1)} & 1 & p_{2} & p_{2}^{2} & \cdots & p_{2}^{n-1} \\
\vdots & \vdots & \vdots & \vdots & \ddots & \vdots \\
P_{n+1}^{(1)} & 1 & p_{n+1} & p_{n+1}^{2} & \cdots & p_{n+1}^{n-1}
\end{array}\right)
$$

where we have dropped the dependence in $x$ to obtain a lighter notation. We claim that

$$
\begin{aligned}
\operatorname{det} M_{1} & =(-1)^{n(n+1) / 2} \prod_{1 \leq i<j \leq n+1}\left(p_{i}-p_{j}\right) \\
& =(-1)^{n(n+1) / 2} \prod_{1 \leq i<j \leq n+1} c_{i, j} \in \mathbb{R} .
\end{aligned}
$$

We prove the claim (12) by induction over the dimension $n \geq 2$ of the matrix $M_{1}$. Assume $n=2$. Then $M_{1}$ becomes the $3 \times 3$ matrix

$$
\left(\begin{array}{lll}
p_{2} p_{3} & 1 & p_{1} \\
p_{1} p_{3} & 1 & p_{2} \\
p_{2} p_{3} & 1 & p_{3}
\end{array}\right)=-\left(p_{1}-p_{2}\right)\left(p_{1}-p_{3}\right)\left(p_{2}-p_{3}\right)=-c_{1,2} c_{1,3} c_{2,3} \in \mathbb{R}
$$


Now we assume it is true for $n=1, \ldots, m-1$ and we will prove it for $n=m$ (obtaining an $(m+1) \times(m+1)$-matrix $\left.M_{1}\right)$. Let $f_{i}$ be the $i$ th row. We subtract to each row $f_{i}$ for $i=2, \ldots, m$, the first row $f_{1}$ and we get that

$$
M_{1}=\left(\begin{array}{cccccc}
P_{1}^{1} & 1 & p_{1} & p_{1}^{2} & \cdots & p_{1}^{m-1} \\
\left(p_{1}-p_{2}\right) P_{1,2}^{1} & 0 & p_{2}-p_{1} & p_{2}^{2}-p_{1}^{2} & \cdots & p_{2}^{m-1}-p_{1}^{m-1} \\
\vdots & \vdots & \vdots & \vdots & \ddots & \vdots \\
\left(p_{1}-p_{m+1}\right) P_{1, m+1}^{1} & 0 & p_{m+1}-p_{1} & p_{m+1}^{2}-p_{1}^{2} & \cdots & p_{m+1}^{m-1}-p_{1}^{m-1}
\end{array}\right)
$$

Let $c_{j}$ be the $i$-th column. We multiply the column $c_{i}$ by $-p_{1}(x)$ and we add it to the column $c_{i+1}$ for $i=3, \ldots, m+1$. Then $M_{1}$ is equal to

$$
\left(\begin{array}{cccccc}
P_{1}^{1} & 1 & p_{1} & 0 & \cdots & p_{1}^{m-1} \\
\left(p_{1}-p_{2}\right) P_{1,2}^{1} & 0 & p_{2}-p_{1} & p_{2}\left(p_{2}-p_{1}\right) & \cdots & p_{2}^{m-2}\left(p_{2}-p_{1}\right) \\
\vdots & \vdots & \vdots & \vdots & \ddots & \vdots \\
\left(p_{1}-p_{m+1}\right) P_{1, m+1}^{1} & 0 & p_{m+1}-p_{1} & p_{m+1}\left(p_{m+1}-p_{1}\right) & \cdots & p_{m+1}^{m-2}\left(p_{m+1}-p_{1}\right)
\end{array}\right)
$$

Therefore, by the Laplace theorem for determinants we obtain that $\operatorname{det} M_{1}=-\operatorname{det} \tilde{M}_{1}$ where $\tilde{M}_{1}$ is the $m \times m$ matrix

$$
\left(\begin{array}{ccccc}
\left(p_{1}-p_{2}\right) P_{1,2}^{1} & p_{2}-p_{1} & p_{2}\left(p_{2}-p_{1}\right) & \cdots & p_{2}^{m-2}\left(p_{2}-p_{1}\right) \\
\vdots & \vdots & \vdots & \ddots & \vdots \\
\left(p_{1}-p_{m+1}\right) P_{1, m+1}^{1} & p_{m+1}-p_{1} & p_{m+1}\left(p_{m+1}-p_{1}\right) & \cdots & p_{m+1}^{m-2}\left(p_{m+1}-p_{1}\right)
\end{array}\right)
$$

Clearly,

$$
\operatorname{det} M_{1}=-\operatorname{det} \tilde{M}_{1}=\prod_{j=2}^{m+1}\left(p_{j}-p_{1}\right) \bar{M}_{1}=(-1)^{m} \prod_{j=2}^{m+1}\left(p_{1}-p_{j}\right) \bar{M}_{1}
$$

where

$$
\begin{aligned}
\bar{M}_{1} & =\left(\begin{array}{cccccc}
P_{1,2}^{1} & 1 & p_{2} & p_{2}^{2} & \cdots & p_{2}^{m-2} \\
\vdots & \vdots & \vdots & \vdots & \ddots & \vdots \\
P_{1, m+1}^{1} & 1 & p_{m+1} & p_{m+1}^{2} & \cdots & p_{m+1}^{m-2}
\end{array}\right) \\
& =\left(\begin{array}{cccccc}
P_{2}^{2} & 1 & p_{2} & p_{2}^{2} & \cdots & p_{2}^{m-2} \\
\vdots & \vdots & \vdots & \vdots & \ddots & \vdots \\
P_{m+1}^{2} & 1 & p_{m+1} & p_{m+1}^{2} & \cdots & p_{m+1}^{m-2}
\end{array}\right)
\end{aligned}
$$

where it is again a $m \times m$ matrix $M_{1}$ (starting in $p_{2}$ instead of $p_{1}$ ). By the induction hypotheses we have that $\operatorname{det} \bar{M}_{1}=\prod_{2 \leq i<j \leq m}\left(p_{i}-p_{j}\right)$ and so

$$
\begin{aligned}
\operatorname{det} M_{1} & =(-1)^{m} \prod_{j=2}^{m+1}\left(p_{1}-p_{j}\right)(-1)^{(m-1) m / 2} \prod_{2 \leq i<j \leq m}\left(p_{i}-p_{j}\right) \\
& =(-1)^{m(m+1) / 2} \prod_{1 \leq i<j \leq m+1}\left(p_{i}-p_{j}\right) \\
& =(-1)^{m(m+1) / 2} \prod_{1 \leq i<j \leq m+1} c_{i, j}
\end{aligned}
$$

which proves (12). 
Now we want to compute the inverse of $M_{1}$. We compute the first row of the matrix $M_{1}^{-1}=\left(m_{i, j}^{-1}\right)_{1 \leq i \leq j \leq n}$, and we will show that $\left(m_{1, j}^{-1}\right)_{1 \leq j \leq n}$ is equal to

$$
\frac{1}{\prod_{1 \leq k \leq n, k \neq j}\left(p_{j}-p_{k}\right)}
$$

Indeed, note that in view of the fact that the determinant of (10) is the same as the determinant of (12) and by the forms of the matrices $M$ and $M_{1}$, it is clear that $\left(m_{1, j}^{-1}\right)_{1 \leq j \leq n}$ is equal to $\left(m_{j, n}\right)_{1 \leq j \leq n}$.

It follows from (13) that

$$
\begin{aligned}
\bar{A}_{0} & =\left(m_{1,1}^{-1}, \ldots, m_{1, n}^{-1}\right) \cdot(1,1, \ldots, 1)^{*} \\
& =\sum_{j=1}^{n} \frac{1}{\prod_{1 \leq k \leq n, k \neq j}\left(p_{j}-p_{k}\right)}
\end{aligned}
$$

Moreover, we claim that

$$
\sum_{j=1}^{n} \frac{1}{\prod_{1 \leq k \leq n, k \neq j}\left(p_{j}-p_{k}\right)}=0
$$

We prove the by induction over the number of elements $n$. If $n=2$ it is clear. Now assume it is true for $n-1$ and we will show it for $n$. We write the left-hand side of (14) as

$$
\begin{aligned}
& \sum_{j=1}^{n} \frac{1}{\prod_{1 \leq k \leq n, k \neq j}\left(p_{j}-p_{k}\right)} \\
& =\frac{1}{p_{n}-p_{n-1}}\left(\sum_{j=1}^{n} \frac{p_{j}-p_{n-1}}{\prod_{1 \leq k \leq n, k \neq j}\left(p_{j}-p_{k}\right)}-\sum_{j=1}^{n} \frac{p_{j}-p_{n}}{\prod_{1 \leq k \leq n, k \neq j}\left(p_{j}-p_{k}\right)}\right)
\end{aligned}
$$

Each of the sums in (15) now has the form of the original sum in (14), except on $n-1$ elements, and the values turn out nicely by induction. Indeed,

$$
\sum_{j=1}^{n} \frac{p_{j}-p_{n}}{\prod_{1 \leq k \leq n, k \neq j}\left(p_{j}-p_{k}\right)}=\sum_{j=1}^{n-1} \frac{1}{\prod_{1 \leq k \leq n-1, k \neq j}\left(p_{j}-p_{k}\right)}
$$

and

$$
\sum_{j=1}^{n} \frac{p_{j}-p_{n-1}}{\prod_{1 \leq k \leq n, k \neq j}\left(p_{j}-p_{k}\right)}=\sum_{j=1, j \neq n-1}^{n} \frac{1}{\prod_{1 \leq k \leq n, k \notin\{j, n-1\}}\left(p_{j}-p_{k}\right)}
$$

and both are zero by induction hypotheses.

Hence, in view of (14) we have that $\bar{A}_{0}=0$. However, then $A_{0}(x)=0$, which is not possible and the proof of statement (f) follows.

Statement (g) is an immediate consequence of statements (e), (f) and (d). The proof of Theorem 1 is complete.

\section{Proof of Theorem 3}

Let

$$
y d y / d x=\varphi^{3} \bar{A}_{0}(x)+A_{1}(x) y+\ldots+A_{n}(x) y^{n}:=F(x, y)
$$

and assume that $y=\varphi(x)$ is a periodic solution. We make the change of variables $z=y-\varphi(x)$ and we obtain

$$
\frac{d z}{d x}=\frac{F(x, z+\varphi(x))}{z+\varphi(x)}-\frac{F(x, \varphi(x))}{\varphi(x)} .
$$

Please note that 


$$
\begin{aligned}
F(x, \varphi(x)) & =\varphi^{3} \bar{A}_{0}(x)+A_{1}(x) \varphi+\ldots+A_{n}(x) \varphi^{n} \\
& =\varphi\left(\varphi^{2} \bar{A}_{0}(x)+A_{1}(x)+\ldots+A_{n}(x) \varphi^{n-1}\right)
\end{aligned}
$$

and so $\frac{F(x, \varphi(x))}{\varphi(x)}$ is always well defined.

With the change of variables $z=y-\varphi(x)$, the periodic solution $y=\varphi(x)$ becomes the solution $z=0$ (is constant and so it is periodic). Please note that

$$
\frac{d z}{d x}=\frac{\varphi^{3} \bar{A}_{0}}{z+\varphi}-\varphi^{2} \bar{A}_{0}(x)+A_{2} z+A_{3}\left[(z+\varphi)^{2}-\varphi^{2}\right]+\ldots+A_{n}\left[(z+\varphi)^{n-1}-\varphi^{n-1}\right]
$$

The right-hand side of Equation (16) is 0 when $z=0$. Set $\psi\left(x ; z_{0}\right)$ to be the solution of Equation (16) with initial condition $z_{0}$. Please note that $\psi(x ; 0)=0$. We want to study the solutions of Equation (16) around $z=0$. We consider the translation operator $\Psi\left(z_{0}\right)=\psi\left(1 ; z_{0}\right)-\psi\left(0 ; z_{0}\right)=\psi\left(1 ; z_{0}\right)-z_{0}$ and we study it near $z_{0}=0$. If $\Psi\left(z_{0}\right)$ is identically zero then the periodic solution belongs to a continuum of periodic solutions. If $\Psi\left(z_{0}\right)$ is not identically zero then the multiplicity of $z=0$ as a limit cycle of the differential equation is the multiplicity of $z_{0}=0$ as a zero of $\Psi\left(z_{0}\right)$ which, by the change of variables, is the multiplicity of $y=\varphi(x)$ for the initial Equation (5).

We expand $\psi\left(x ; z_{0}\right)$ in Taylor series around $z_{0}=0$ and we get

$$
\psi\left(x ; z_{0}\right)=h_{1}(x) z_{0}+h_{2}(x) z_{0}^{2}+h_{3}(x) z_{0}^{3}+O\left(z_{0}^{4}\right),
$$

where $h_{i}(x)$ are differentiable functions for $i=1,2,3$ and $h_{1}(0)=1$ while $h_{2}(0)=h_{3}(0)=0$, because $\psi\left(0 ; z_{0}\right)=z_{0}$. We have that $\psi\left(x ; z_{0}\right)$ satisfies the equality

$$
\begin{aligned}
\frac{\partial \psi\left(x ; z_{0}\right)}{\partial x}= & \frac{\varphi^{3} \bar{A}_{0}}{\psi\left(x ; z_{0}\right)+\varphi}-\varphi^{2} \bar{A}_{0}(x)+A_{2} \psi\left(x ; z_{0}\right)+A_{3}\left[\left(\psi\left(x ; z_{0}\right)+\varphi\right)^{2}-\varphi^{2}\right] \\
& +\ldots+A_{n}\left[\left(\psi\left(x ; z_{0}\right)+\varphi\right)^{n-1}-\varphi^{n-1}\right] \\
= & \frac{\varphi^{3} \bar{A}_{0}}{\psi\left(x ; z_{0}\right)+\varphi}-\varphi^{2} \bar{A}_{0}(x)+\mathcal{F}\left(x, \psi\left(x ; z_{0}\right)+\varphi\right)-\mathcal{F}(x, \varphi),
\end{aligned}
$$

where $\mathcal{F}(x, y)$ was introduced in (2). Now we expand this equation in Taylor series around $z_{0}=0$. Since this identity must hold for any value of $z_{0}$ in the neighborhood around $z_{0}=0$, the coefficients of the same powers of $z_{0}$ must be equal. Hence, we obtain the following system of differential equations for the functions $h_{i}(x)$ for $i=1,2,3$ (we dropped the dependency of $h_{i}, \varphi, \bar{A}_{0}$ in $x$ for simplicity of the notation):

$$
\begin{aligned}
h_{1}^{\prime}= & -\left(\varphi^{3} \bar{A}_{0}-\frac{\partial \mathcal{F}}{\partial y}(x, \varphi)\right) h_{1}, \\
h_{2}^{\prime}= & -\left(\varphi^{3} \bar{A}_{0}-\frac{\partial \mathcal{F}}{\partial y}(x, \varphi)\right) h_{2}+\left(\varphi^{2} \bar{A}_{0}+\frac{1}{2} \frac{\partial^{2} \mathcal{F}}{\partial y^{2}}(x, \varphi)\right) h_{1}^{2}, \\
h_{3}^{\prime}= & -\left(\varphi^{3} \bar{A}_{0}-\frac{\partial \mathcal{F}}{\partial y}(x, \varphi)\right) h_{3}+\left(2 \varphi^{2} \bar{A}_{0}+\frac{\partial^{2} \mathcal{F}}{\partial y^{2}}(x, \varphi)\right) h_{1} h_{2} \\
& -\left(\varphi \bar{A}_{0}-\frac{1}{6} \frac{\partial^{3} \mathcal{F}}{\partial y^{3}}(x, \varphi)\right) h_{1}^{3} .
\end{aligned}
$$

The solution of the system in (17) satisfies

$$
\begin{aligned}
& h_{1}(x)=e^{\mathcal{A}_{1}(x)} \\
& h_{2}(x)=h_{1}(x) \mathcal{A}_{2}(x), \\
& h_{3}(x)=h_{1}(x) \mathcal{A}_{3}(x)+\frac{h_{2}(x)^{2}}{h_{1}(x)}
\end{aligned}
$$


where the functions $\mathcal{A}_{i}(x)$ for $i=1, \ldots, 3$ are the ones provided in the statement of the theorem. We observe that $h_{1}(x)>0$ for any $x$ and so, the former expressions are well defined. These expressions yield that $h_{1}(1)=1$ if and only if $\mathcal{A}_{1}(1)=0$. In addition, for fixed $k \in\{2,3\}$, we have $h_{1}(1)=1$ and $h_{i}(1)=0$ for all $i$ such that $2 \leq i \leq k$ if and only if, $\mathcal{A}_{i}(1)=0$ for all $i$ such that $1 \leq i \leq k$.

The translation operator reads as

$$
\Psi\left(z_{0}\right)=\left(h_{1}(1)-1\right) z_{0}+h_{2}(1) z_{0}^{2}+h_{3}(1) z_{0}^{3}+O\left(z_{0}^{4}\right),
$$

and so $z_{0}=0$ is a limit cycle of multiplicity $m \in\{1,2\}$ if and only if $\mathcal{A}_{i}(1)=0$ for all $i$ such that $1 \leq i<m$ and $\mathcal{A}_{m}(1) \neq 0$.

Finally, if $\mathcal{A}_{1}(1)=\mathcal{A}_{2}(1)=\mathcal{A}_{3}(1)=0$, then either $z=0$ is a limit cycle of multiplicity greater than or equal to 4 , or it belongs to a continuum of periodic solutions. This concludes the proof of the theorem.

\section{Conclusions}

Theorem 1 provides very accurate universal bounds (in the sense that they only depend on the degree $n$ on the $y$-variable) on the number of polynomial solutions of Equation (1) and such bounds are optimal. Theorem 3 considers the multiplicity of the differential Equation (1) and shows that the problem is somehow related with the 16th's Hilbert problem. Similar techniques used in the paper could be applied to obtain upper bounds on the number of polynomial solutions of equations of the type (1) in which the left-hand side is a power of $y$ (or even a polynomial in $y$ ) but the complexity grows substantially especially if one wants to obtain accurate upper bounds (and that such bounds are reached).

Funding: Partially supported by FCT/Portugal through the project UID/MAT/04459/2019.

Conflicts of Interest: The author declares no conflict of interest.

\section{References}

1. Rainville, E.D. Necessary conditions for polynomial solutions of certain Riccati equations. Am. Math. Mon. 1936, 43, 473-476. [CrossRef]

2. Campbell, J.G.; Colomb, M. On the polynomial solutions of a Riccati equation. Am. Math. Mon. 1954, 61, 402-404. [CrossRef]

3. Behoul, D.; Cheng, S.S. Computation of all polynomial solutions of a class of nonlinear differential equations. Computing 2006, 77, 163-177. [CrossRef]

4. Ferragut, A.; Llibre, J. On the polynomial solutions of the polynomial differential equations $y y^{\prime}=a_{0}(x)+$ $a_{1}(x) y+a_{2}(x) y^{2}+\cdots+a_{n}(x) y^{n}$. Indian J. Pure Appl. Math. 2020, 51, 217-232. [CrossRef]

5. Roussarie, R. Bifurcations of planar vector fields and Hilbert's sixteenth problem. In Progress in Mathematics; Birkhauser: Basel, Switzerland, 1998; Volume 164.

6. Álvarez, M.J.; Bravo, J.L.; Fernández, M. The number of limit cycles for generalized Abel equations with periodic coefficients of definite sign. Commun. Pure Appl. Anal. 2009, 8, 1493-1501. [CrossRef]

7. Álvarez, M.J.; Bravo, J.L.; Fernández, M. Existence of non-trivial limit cycles in Abel equations with symmetries. Nonlinear Anal. 2013, 84, 18-28. [CrossRef]

8. Álvarez, M.J.; Bravo, J.L.; Fernández, M. Limit cycles of Abel equations of the first kind. J. Math. Anal. Appl. 2015, 423, 734-745. [CrossRef]

9. Álvarez, M.J.; Bravo, J.L.; Fernández, M.; Prohens, R. Centers and limit cycles for a family of Abel equations. J. Math. Anal. Appl. 2017, 453, 485-501. [CrossRef]

10. Álvarez, M.J.; Gasull, A.; Yu, J. Lower bounds for the number of limit cycles of trigonometric Abel equations. J. Math. Anal. Appl. 2008 ,342, 682-693. [CrossRef]

11. Fossas, E.; Olm, J.M.; Sira-Ramírez H. Iterative approximation of limit cycles for a class of Abel equations. Physica D 2008, 237, 3159-3164. [CrossRef] 
12. Gasull, A.; Llibre, J. Limit cycles for a class of Abel equations. SIAM J. Math. Anal. 1990, 21, 1235-1244. [CrossRef]

13. Ilyashenko, Y. Hilbert-type numbers for Abel equations, growth and zeros of holomorphic functions. Nonlinearity 2000, 13, 1337-1342. [CrossRef]

14. Yamaleev, R.M. Representation of solutions of $n$-order Riccati equation via generalized trigonometric functions. J. Math. Anal. Appl. 2014, 420, 334-347. [CrossRef]

15. Yamaleev, R.M. Solutions of Riccati-Abel equation in terms of third order trigonometric functions. Indian J. Pure Appl. Math. 2014, 45, 165-184. [CrossRef]

16. Álvarez, M.J.; Bravo, J.L.; Fernández, M.; Prohens, R. Alien limit cycles in Abel equations. J. Math. Anal. Appl. 2020, 482, 123525. [CrossRef]

17. Cima, A.; Gasull, A.; Manõsas, F. On the number of polynomial solutions of Bernouilli and Abel polynomial differential equations. J. Differ. Equ. 2017, 263, 7099-7122. [CrossRef]

18. Gasull, A.J.; Torregrosa, J.; Zhang, X. The number of polynomial solutions of polynomial Riccati equations. J. Differ. Equ. 2016, 261, 5071-5093. [CrossRef]

19. Giné, J.; Grau, M.; Llibre, J. On the polynomial limit cycles of polynomial differential equations. Isr. J. Math. 2011, 181, 461-475. [CrossRef]

20. Valls, C. Rational limit cycles on Bernouilli and Riccati equations. J. Geom. Phys. 2020, 155, 103705. [CrossRef]

21. Liu, C.; Li, C.; Wang, X.; Wu, J. On the rational limit cycles of Abel equations. Chaos Solitons Fractals 2018, 110, 28-32. [CrossRef]

22. Valls, C. Rational limit cycles on Abel polynomial equations. Mathematics 2020, 8, 885. [CrossRef]

23. Poznyak, A.S. Advanced Mathematical Tools for Automatic Control Engineers; Elsevier B.V.: Amsterdam, The Netherlands, 2008; pp. xxviii+774.

24. Lloyd, N.G. The number of periodic solutions of the equation $\dot{z}=z^{N}+p_{1}(t) z^{N-1}+\ldots+p_{N}(t)$. Proc. Lond. Math. Soc. 1973, 27, 667-700. [CrossRef]

25. Lloyd, N.G. A note on the number of limit cycles of certain two-dimensional systems. J. Lond. Math. Soc. 1979, 20, 277-286. [CrossRef]

(C) 2020 by the author. Licensee MDPI, Basel, Switzerland. This article is an open access article distributed under the terms and conditions of the Creative Commons Attribution (CC BY) license (http:/ / creativecommons.org/licenses/by/4.0/). 